\title{
Development of Wireless Remote Patient Monitoring System using ARM 7 and LabVIEW
}

\author{
Gulab M. Dhakne ${ }^{1}$, Satvashil N. Gaikwad ${ }^{2}$ \\ M.E. Student, Dept of Electronics and Telecommunication Engineering, Deogiri Institute of Engineering and \\ Management Studies, Aurangabad (M.S.), India ${ }^{1}$ \\ Asst. Professor, Dept of Electronics and Telecommunication Engineering, Deogiri Institute of Engineering and \\ Management Studies, Aurangabad (M.S.), India ${ }^{2}$
}

\begin{abstract}
Electronics is the diverse field of engineering finding applications in almost all areas of human life. One of the most important applications is in biomedical engineering. Recent medical sciences and association claims that the number of the patients suffering from heart diseases increasing day by day. This might be due to changing life style, work load, over tension, competition, lack of exercise etc. So timely monitoring of such a patient's heart activities like heartbeat, ECG is in demand now days. This further increases rush in the hospitals and increases patient expenditure also. This encourage us to design such a system which can monitor patient at his home place continuously or whenever required and all the body parameters could be shared by the doctor at hospital in real time. Whenever any parameter value exceeds normal range then SMS is also sent to the doctor thereafter rapid treatment can be provided to the patient. This project used ARM 7 LPC 2148 development board. Patients body temperature, heart beat and ECG are acquired from body through various sensors, processed it and displayed on LCD as well as on NI LabVIEW through HC 05 Bluetooth module. It is then transmitted to doctors PC using team viewer which provides connectivity in both sides. SIM 900 GSM/GPRS Module is used for sending the SMS. The objective of this project is to design a configuration system which will require optimum power for its operation and should be cost efficient.
\end{abstract}

Keywords: SIM 900, GSM/GPRS, NI LabVIEW, ECG, LUT.

\section{INTRODUCTION}

As discussed earlier electronics technology is finding many applications in medical field and becoming popular in wireless remote patient monitoring which is in demand now days. The design purpose of our project is to monitor the parameters of patient's heart activity such as ECG, pulse rate along with body temperature wirelessly from a patient's location to the doctors section at hospital where investigations of this data could be achieved by cardiologist.

Due to shortage of resources i.e. hospitals and physicians which does not meet with the requirement of tremendous increase in population of India. It is needed to healthcare professional to use electronics equipment's for monitoring and diagnosis of patient's data from outside of the hospital. This problem of shortage of resources and physicians becomes a challenge to the system which needs to provide the ways for workload easing so that many patients diagnosis and monitoring can be achieved simultaneously. This challenge encouraged the electronics technocrats and engineers to design and develop advance technology in biomedical field. Heart rate measurement can be done either by using pulse method which is carried out by examining the flow of blood into the finger or by the ECG waveform. The pulse method is simple and convenient. During the hearts systolic stroke blood flows into the body parts and into the finger via the radial artery on the arm. This flow of blood into the finger can be sensed photo electrically using IR sensor. Heart rate is detected when light intensity changes as blood flows into the finger.

Early day's tape recording method was used for diagnosis for heart disease which typically based on of Electro Cardio Gram (ECG) signal which is then studied and analyzed using a microcomputer. In this method, the real time heart rate is measured by placing the finger in the IR sensor. At the same time, after the processing of heart rate any diseases can be detected such as, bradycardia or tachycardia either for adult or children.

The ECG module is used to detect and plot the tiny potential changes in the heart which generates during atrial depolarization, ventricular depolarization and ventricular repolarization. The impulse generates in the Sinoatrial (SA) node and transmits to the Atrioventricular (AV) node as shown in figure 1 . The one heart beat corresponds to the one ECG waveform. The time period of ECG waveform reduces as heart rate increases and vice versa.

Figure 1 shows the $\mathrm{P}, \mathrm{Q}, \mathrm{R}, \mathrm{S}$ and $\mathrm{T}$ waves on an electrocardiogram tracing (lead 1) illustrating the three normally recognizable deflection waves and the important intervals. 
Vol. 5, Issue 12, December 2016

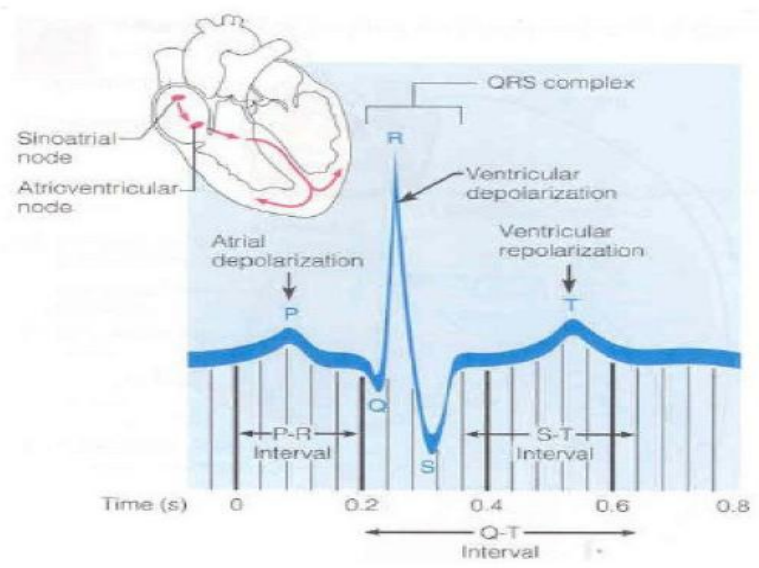

Fig. 1: The Electrocardiogram (ECG) plot

The objective over designing of this system is to provide solutions to the problems which encountered during the transmission of ECG and other body parameters from a location to a remote site where this data could be investigated by a cardiologist. As like pulse method the patient's heart beat can also be measured by using the waveform of ECG. The pulse method for heart beat detection is easy and convenient.

Many researches have done on patient monitoring. Telemedicine and telemonitoring have been used in several researches. Telemonitoring network is dedicated to medical teleservices; the people who live in isolated and rural areas are the main focus of it [1].

This system continuously aware and update doctor regarding health status of patient. Through this way physicians can also provide best health care after the diagnosis of proper disease. Hence it provides comfort to the patients, reducing rush in the hospitals thus saving time and money of the patient.

In this Project the solution is provided to the problem which occurs during transmission of patient monitoring data such as pulse rate, ECG \& temperature from a patient's remote site location to the hospital where this data could be monitored continuously by the cardiologist.

This work mainly focuses on design and implementation of remote patient monitoring system in which continuous monitoring of the patient's body parameters is achieved wirelessly for a patient who is situated in his home or remote place and not in reach of hospital and inform and aware the doctor regarding any abnormal and fatal parameters values by sending SMS, so that rapid treatment can be provided to the patient.

The system described here is a microcontroller-based portable system for diagnosis and controlling of heart rate, ECG and temperature on real time. The ECG signal recording using tape recording systems are large in size and prone to mechanical failure and it needs large batteries also. In order to reduce the weight, size and power consumption of the system, a single chip Reduced Instruction Set Computer (RISC) architecture ARM 7 family LPC 2148 microcontroller is chosen. The AD 8232
ECG sensor is used to examine the heart activity. LM 358 based IR heart beat sensor is used to provide pulse rate from body by placing the finger into the IR sensor to the Analog to Digital converter circuit which is mounted on LPC 2148 microcontroller. LM 35 Precision Centigrade Temperature Sensors is used to measure body temperature. $16 * 2$ liquid crystal display is used to display the temperature in Fahrenheit, pulse rate in beats per minutes and ECG values.

Different types of electrodes and sensors are used which is placed on desired locations of the patient's body to extract the raw data, filtered it and transmitted wirelessly through Bluetooth module to display graphically using LabVIEW software. The continuous recorded real time data is then shared with the doctor using team viewer to examine the ECG signals and further guides the patient for any emergency medical aid required.

\section{LITERATURE SURVEY}

Different ECG Monitoring system architecture are discussed in this section

Several similar methods available for patient monitoring applications are discussed: The system discussed in [2] presents a mixed-signal ECG SoC, which uses integrated AFE analog front-end and DSP back-end. Analog front end supports concurrent 3-channel electrodes for monitoring of ECG, with impedance measurement and band-power extraction. The system describes method for reduction of motion artifacts, accurate detection of the $R$ peak analysis of HRV etc. The system with webpage applications is discussed in [3]. It consists of three subsystems including patient subsystem, web server and database subsystem and android unit subsystem. In patient unit subsystem electrodes are used for acquisition of electrical activity and bio potential signals of heart, instrumentation amplifier is used for amplification of signal and transmission of signal to patients PC in several time intervals. ECG data is stored and publishing of abnormal result is achieved in Web Server and Database subsystem. Only person with authority can access the data. Here database is implemented and detection of abnormality is achieved. The doctor use android based applications to access the details of patients using smartphone. ECG Note for doctor and My Note for patient these two applications are developed.

The implementation of system in [4] is with the help of specially designed ECG amplifier and a power efficient microcontroller eZ430-RF2500 board designed by Texas instruments. The personal server is used to receive the information wirelessly from designed module of ECG. Wearable device for wireless transmission of ECG is proposed in [5], [6] where LMS algorithm based adaptive filters are used to reduce motion artifact. Also MAC unit in the LMS core is replaced using LUT. Optimization of this LUT is achieved by using APC-OMS technique. It benefits less power consumption and small in size than the 
Vol. 5, Issue 12, December 2016

existing LMS algorithm. An architecture using VLSI is The system is supplied by the $230 \mathrm{~V} / 9 \mathrm{~V}$ transformer developed and implemented for LMS algorithm to design having $750 \mathrm{~mA}$ current supplying capability. The wearable ECG ASIC for reduction of size and power.

transformer supply is provided to the processor, heart beat The configurable and portable ECG monitoring systems in real time is implemented in [7] where ECG data is acquired from the sensor and is transmitted wirelessly using Zigbee-802.15.4 wireless module. This transmitted data is received at the receiver with the same module and then further received by the com port of PC for further processing.

Somehow similar approach for the remote patient monitoring i.e. heartbeat, temperature and ECG is proposed in [8] where IR heart beat sensor and LM 35 temperature sensor is used along with ECG amplifier circuit using instrumentation amplifier. The body parameter values are transmitted wirelessly to the PC using Bluetooth module and displayed on NI LabVIEW. The team viewer is used for sharing of real time data to the doctor.

\section{SYSTEM DESIGN CONCEPT}

The designed system block diagram is shown in figure 2 . The 64 pin IC ARM 7 family LPC 2148 microcontroller board is used which is mostly used for low power application and can be used to process the 16 as well as 32 bit data. The working principle of the system is divided into three parts i.e. hardware description, LabVIEW description and Team viewer.

\section{A. Hardware Illustration and Working}

The interfacing diagram for developed system is shown in figure 3. A 64 pin IC ARM 7 family's LPC 2148 processor is used as a heart of the system. The LPC 2148 board contains RESET and ISP (In System Programming) switches. To dump the program into the system first press the ISP button and press and release RESET button then program will dump into the system through port 0 .
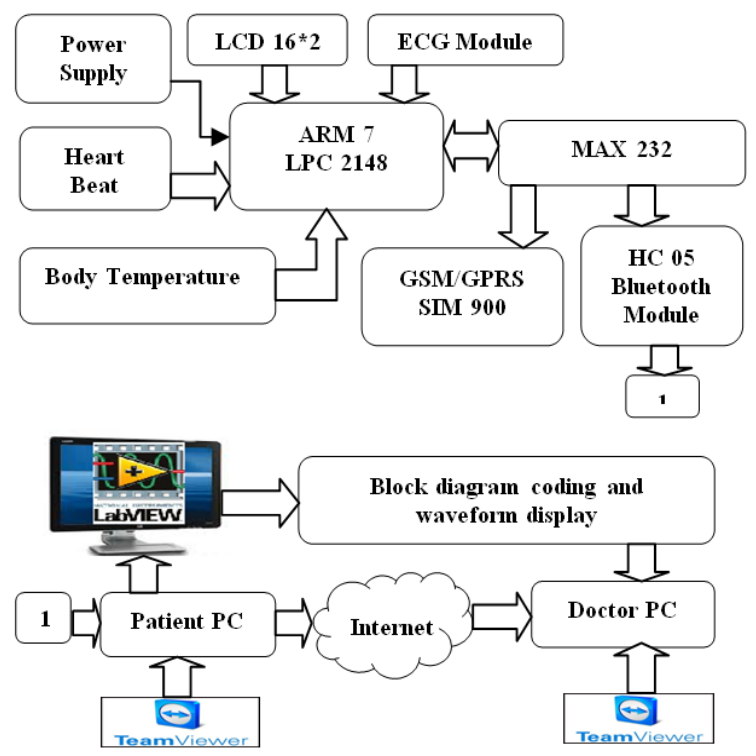

Fig. 2 Block diagram of designed system

two $5 \mathrm{~V}$ adaptor. Here two ports UART 0 and UART 1 are used. UART 0 is connected to the Bluetooth module and UART 1 to the SIM 900 GSM/GPRS module. MAX 232 voltage level converter IC is used to for matched stabilization purpose. For temperature measurement LM 35 precision centigrade temperature sensor is used which can measure the temperature of atmosphere and patient body as well if placed properly on a body. LM 35 is interfaced to the pin P0.28.

Here ECG module AD 8232 is selected which consists of three electrodes which are connected to the Right Arm (RA), Left Arm (LA) and Right Leg (RL) on the body of the patient who's ECG has to be examined. The output of

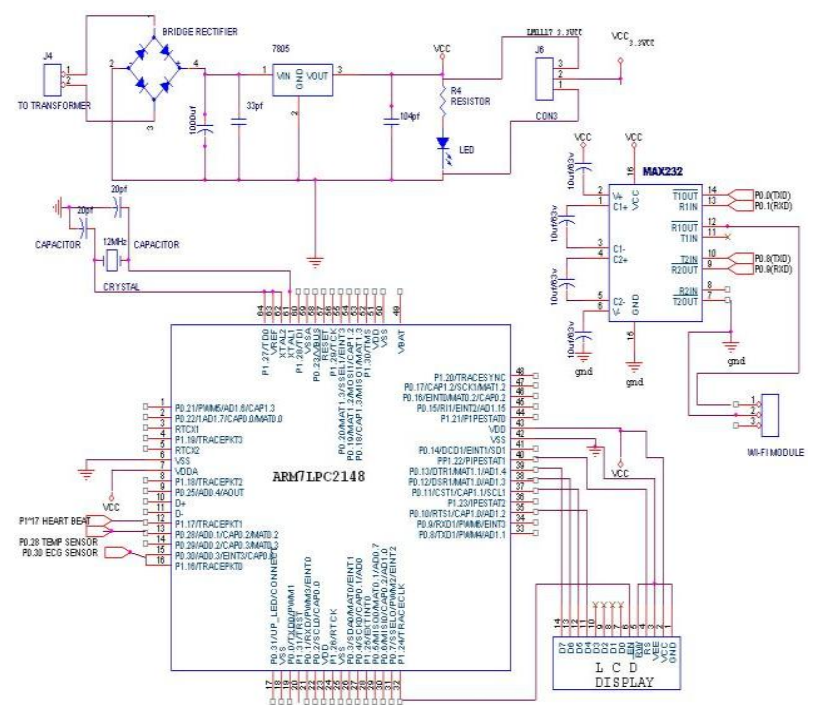

Fig. 3 System interfacing diagram

IR sensor output is provided to the LM 358. After amplification high low pulses are provided to pin number P1.17. Number of pulses are detected in 10 seconds which is then multiplied by 6 to give number of pulses per minute i.e. beats per minutes (BPM).

ECG module is connected to the ADC port P0.28 third channel. The electrical signal generated at sinoatrial (SA) node and travels towards Atrioventricular (AV) node, in this duration one ECG wave having $\mathrm{P}, \mathrm{Q}, \mathrm{R}, \mathrm{S}, \mathrm{T}$ sub waves are generated with specific time interval and amplitude.

The LM 358 based IR heart beat sensor is used to measure the heart beats of the patient which is measured in beats per minutes. The finger is placed in between the LED and Photo detector of the IR sensor which counts the number of beats by interrupting the light ray from LED to photo detector as blood force through the veins during each heartbeat. 


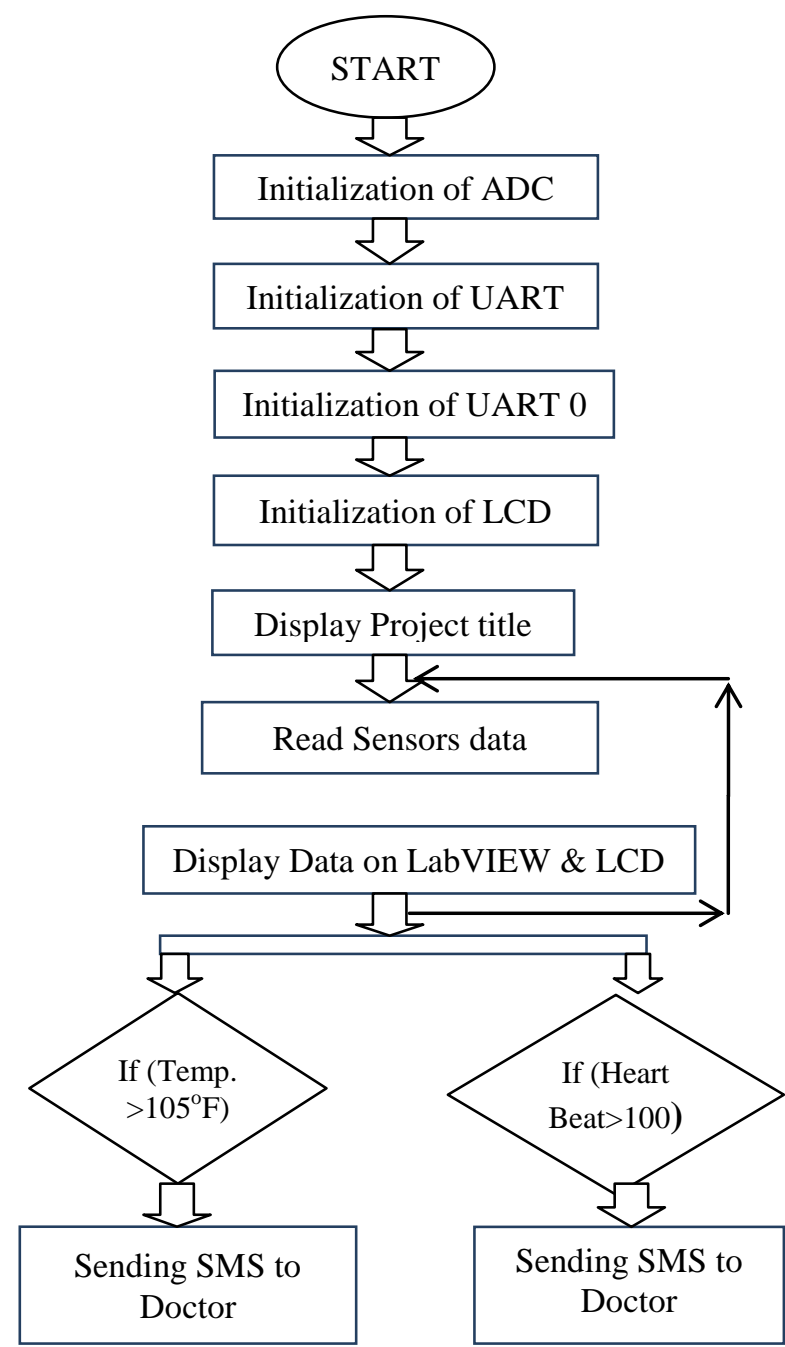

Fig. 4 Flow chart of program

This data is send to the UART 0 to Bluetooth module HC 05 with 9600 baud rate and $2.4 \mathrm{GHz}$ frequency. SIM 900 GSM/GPRS Module is used to send the SMS to doctor when temperature goes above $105^{\circ} \mathrm{F}$ and heart beat goes above 100 beats per minute through UART 1 . The system works according to the instructions given in embedded $\mathrm{C}$ program which is written in Keil uVision4 and dumped into the system using flash magic. The flow chart for the program execution is shown in figure 4.

The body parameters values are extracted from the body with the help of sensors and provided to the ARM 7 LPC 2148 processor after amplification; it is then processed and transmits wirelessly through Bluetooth to the Laptop/PC for display where NI LabVIEW 2013 is installed. The values are also displayed on $16 * 2$ LCD display.

\section{B. LabVIEW Illustration and Working}

National Instruments NI LabVIEW is used in this project for simulation. The LabVIEW block diagram coding is shown in figure 5 . The block coding is performed in NI LabVIEW to display the real time values and waveforms of Temperature, Heart Beat and ECG. The different elements are selected from tool palettes. The data received by the Bluetooth receiver of the Laptop/PC through COM Port transmitted by the Bluetooth transmitter HC 05 .

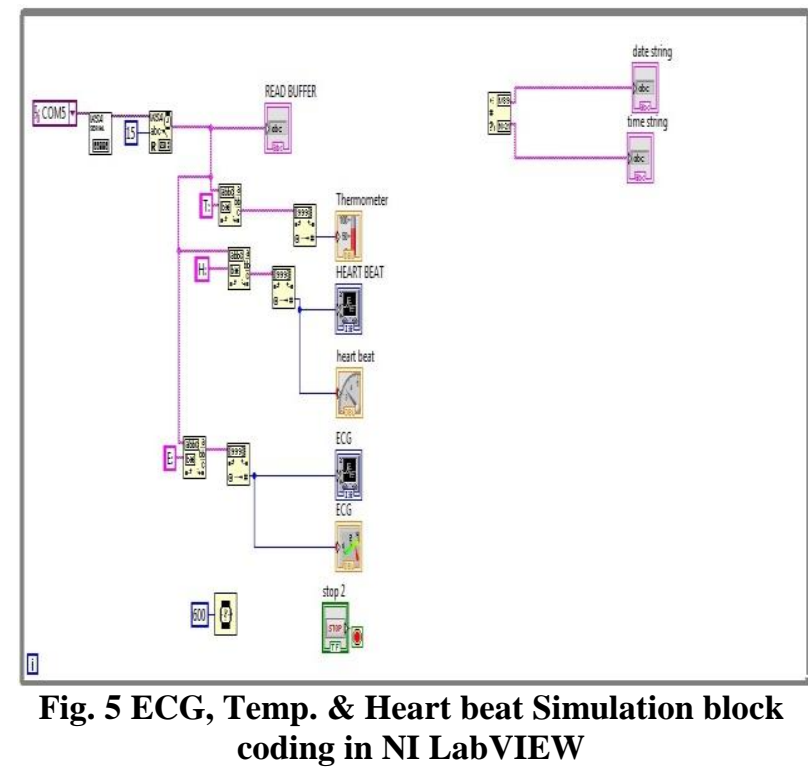

The data through Com port is provided to the VISA (Virtual Instrument Serial Architecture) serial port; it is a string that uniquely identifies the resource to be opened and written to as well as read from. This data is then provided to the VISA configure serial port where length is provided to the data to display which is taken 15 where 15 represent number of characters and here 5 characters are assigned to each parameter for display. For example T: $084 \mathrm{H}: 077 \mathrm{E}: 126$. After the separation of the data it is stored in the buffer. The data is displayed separately on garage and meters after segregation. The flowchart for LabVIEW simulation block coding is shown in figure 6 .

\section{Team Viewer Illustration and Working}

Team Viewer is a proprietary computer software package which can be used for various applications such as desktop sharing, remote control, online meetings, web conferencing and file transfer between computers. The software operates with the Microsoft Windows, Linux, Android, Windows RT and Windows Phone operating systems. It is also possible to access a machine running Team Viewer with a web browser.

While the main focus of this app is remote control of computers, collaboration and presentation features are included. As an aim of this project to remotely monitor the patient from his residential place team viewer is needed. Here it is needed to install the team viewer in both the PC's of patient as well as doctor. To achieve the connectivity in both side internet connections is required. Through this way whatever the values of patients body parameters displaying on the desktop of the patient can be shared by the doctor. Hence continuous monitoring of the patient can be achieved by the doctor remotely and wirelessly. 


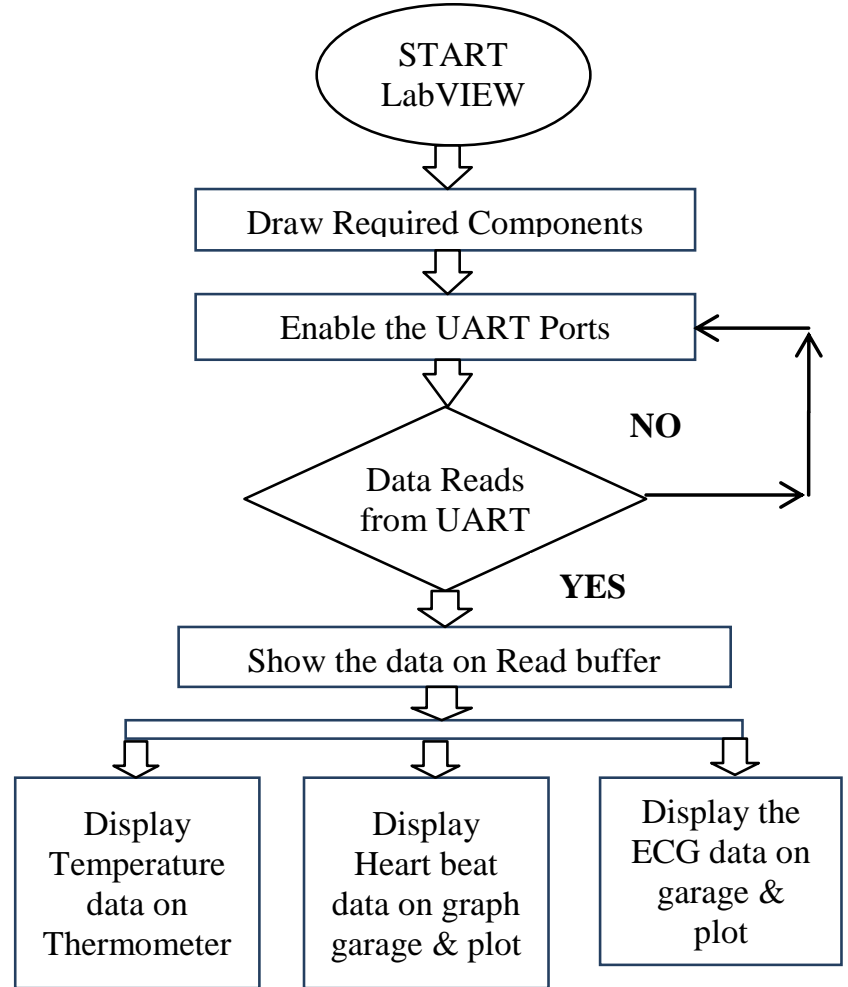

Fig. 6 Flow chart for Lab VIEW simulating code

\section{SENSORS AND HARDWARE TOOLS}

Various sensors are used in the system to extract the body parameters and hardware tools to transmit these data wirelessly to patients PC as well as doctors PC and mobile. The brief review of these sensors is given below.

\section{A. Temperature Sensor}

To acquire the body temperature LM 35 precision centigrade temperature sensor is used. As it draws only 60 $\mu \mathrm{A}$ from its supply, it has very low self-heating, less than $0.1^{\circ} \mathrm{C}$ in still air. The LM35 is rated to operate over a $-55^{\circ}$ to $+150^{\circ} \mathrm{C}$ temperature range.

\section{B. Heart Beat Sensor}

LM 358 based IR heart beat sensor is used to detect the pulse rate of patient. The finger is placed between the LED and photo detector where light intensity is changes as

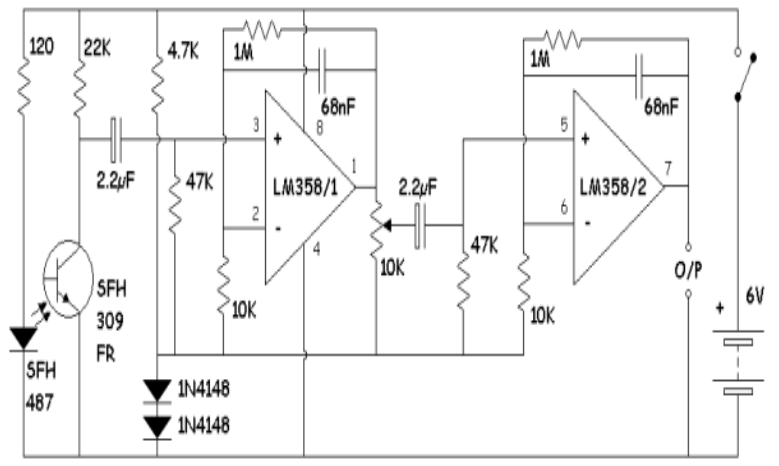

Fig. 7 LM 358 based Heartbeat detector circuit blood circulates through the finger which is detected by the sensor and each change in the light intensity is detected as heartbeat. The circuit diagram of LM 358 based heart beat sensor is shown in figure 7 .

\section{ECG Sensor}

To monitor and plot the hearts cardiac activity of patient AD 8232 ECG sensor is used. Three electrodes are used which are connected to the Right Arm (RA), Left Arm (LA) and Right Leg (RL) on the body of the patient who's ECG has to be examined. The AD8232 is used in applications to measure and conditioned ECG and other bio potential signals as well. It is an integrated signal conditioner. It extracts the electrical signal generated by heart, amplify it and filter in the presence of noisy conditions, which may create due to motion artifacts or improper placement of electrodes. Total 9 connections are available to the AD8232 module. SDN, LO+, LO-, OUTPUT, 3.3V, GND pins are essential to operate with an Arduino, ARM 7 or other development board. Other pins are provided on this module are RA (Right Arm), LA (Left Arm) and RL (Right Leg) to attach and use any custom sensors. There is LED which pulsate during each heartbeat rhythm. The ECG sensor is shown in figure 8.

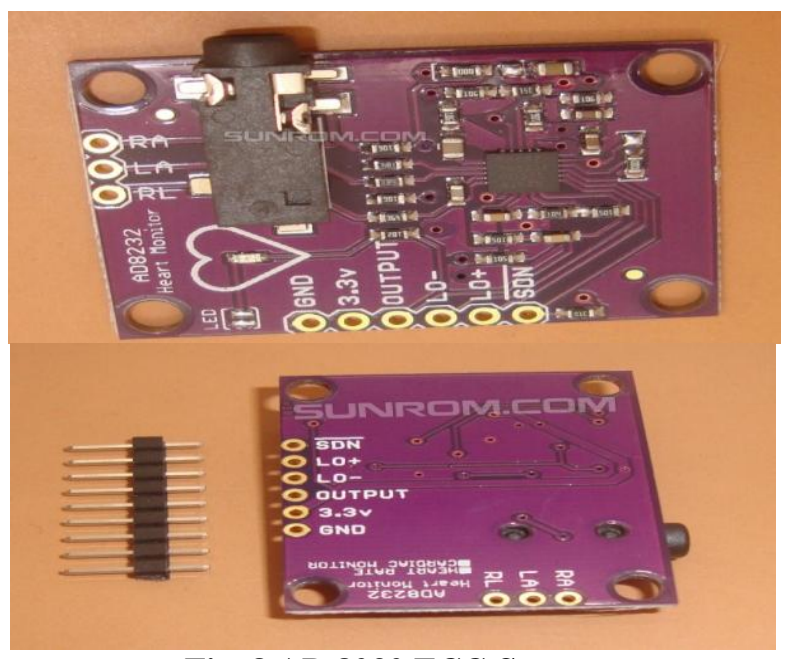

Fig. 8 AD 8232 ECG Sensor

\section{DESIGNED SYSTEM WITH RESULTS}

The figure 9 shows the photograph of designed system. The system results are displayed on LCD in the form of values and it is wirelessly transmitted to the PC with the help of Bluetooth and also displayed in NI LabVIEW 2008 simulating software in real time in the form of values as well as graph. The screenshot for the NI LabVIEW real time waveforms are shown in figure 8 . The voltage of QRS complex generated by the heart is generally from 0.3 to $1 \mathrm{mV}$ which is very small and needs to amplify so that it can drive rest of the instrumentation system and display devices. Hence it is amplified by the ECG sensor AD8232 which can provide RMS values of voltage $V_{\text {rms }}$ up to 1.64 $\mathrm{V}$ and maximum value $\mathrm{V}_{\max }$ up to $2.72 \mathrm{~V}$. One ECG cycle is generated according to the one heart beat i.e. time 
Vol. 5, Issue 12, December 2016

duration of one ECG cycle comprises of 0.8 seconds for a $75 \mathrm{bpm}$ heart beat reading and it changes as heart beat changes. It is found that time period of ECG cycle reduces as heartbeat increases and vice versa. System shows heart beat reading in real time which increases after having exercise. As heartbeat exceeds $100 \mathrm{bpm}$ then SMS is sent to the doctor to aware him about over heartbeat rate.

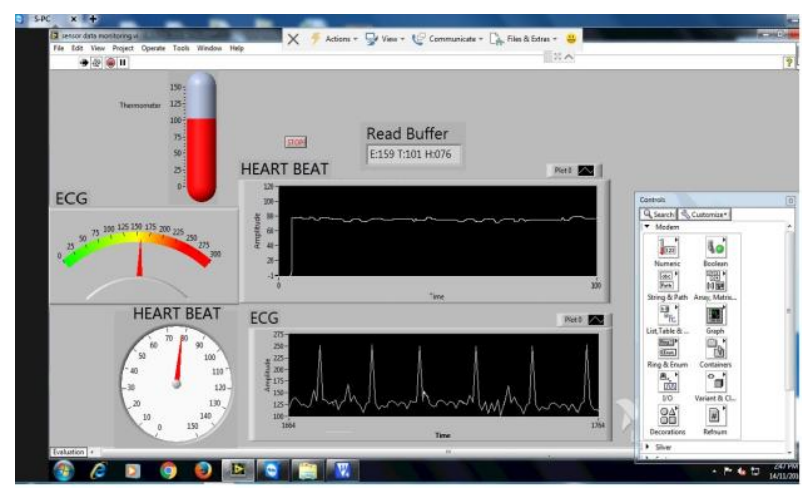

Fig. 9 LabVIEW Simulator showing parameter value \& graphs

LM 35 the precision centigrade temperature sensor in normal condition is measured around $27^{\circ} \mathrm{C}$ with a tolerance of 1 to $1.5^{\circ} \mathrm{C}$. The increase in temperature found for a person suffered from fever and message was sent to the mobile when temperature exceeded $105^{\circ} \mathrm{F}$. The temperature is converted into degree Fahrenheit from degree Celsius by following formula.

$$
\mathrm{T}_{\left({ }^{\circ} \mathrm{F}\right)}=\mathrm{T}_{\left({ }^{\circ} \mathrm{C}\right)} \times 9 / 5+32
$$

This conversion is achieved in the program itself.

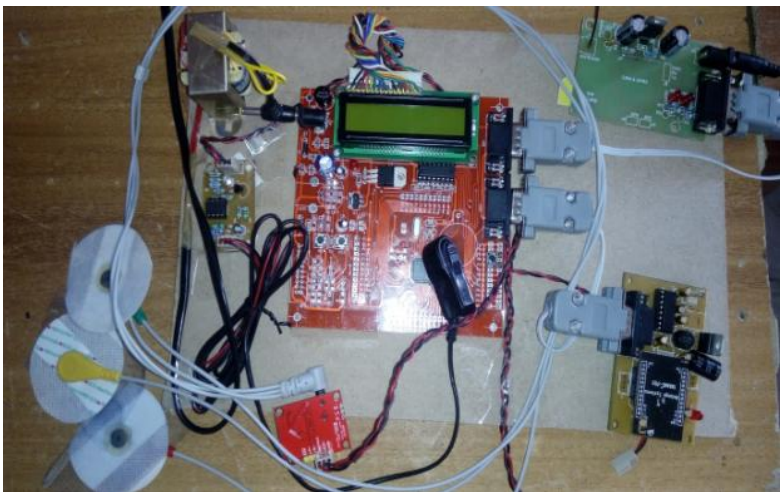

Fig. 10 Designed system photograph

The system is supplied by $230 \mathrm{~V} / 9 \mathrm{~V}$ transformer based power supply having $750 \mathrm{~mA}$ current carrying capability except SIM 900 GSM/GPRS module which is supplied by an adaptor of $5 \mathrm{~V}$ output. Hence system requires less power for its operation i.e. power efficient and also design can be achieved with the help of sensors which costs less hence it is affordable i.e. cost efficient also.

\section{CONCLUSION}

The designed system fulfills the requirement of wireless patient monitoring. The heartbeat, temperature and ECG of the patient can be successively extracted from the body of the patient and transmitted to the doctor who is situated in the hospital. This reduces the rush in the hospitals and thus reducing the patient expenditure also. The system also provide facility to alert the doctor by sending SMS for any abnormal situation i.e. when body parameters exceeds normal values. This further alert the doctor to take immediate action by providing him rapid treatment. In this way patient diagnosis can be achieved. The system uses ARM 7 LPC 2148 microcontroller board which is 64 pin IC advance RISC machine mostly used for low power applications. The other sensors and hardware tools used also consumes less power as it is supplied through $230 \mathrm{~V} / 9 \mathrm{~V}$ transformer based power supply having $750 \mathrm{~mA}$ current carrying capability and 5V adaptor for SIM 900 GSM/GPRS module. As it requires less power for its operation it is power efficient. The communication between hardware and PC can be achieved easily with the help of Bluetooth module.

The program is written in Keil uVision4 software in embedded $\mathrm{C}$ language and dumped into the system using Flash Magic. NI LabVIEW simulator is used to connect the hardware to the PC wirelessly through Bluetooth and display the data and waveform in real time. The real time results i.e. parameters values can be shared with doctors with the help of Team viewer which provides connectivity in both sides providing security also.

\section{REFERENCES}

[1] Anon. Cost of Health Care to reach $\$ 172$ billion: report. CanWest MediaWorks Publications Inc. Ottawa Citizen, November 14, 2008. http://www.canada.com/ottawacitizen/news/story.html?id=c18006b 1-1406-4bbe-9dbfc7edaf652d42

[2] Hyejung Kim, Member, IEEE, Sunyoung Kim, Member, IEEE, Nick Van Helleputte, Member, IEEE, Antonio Artes, Mario Konijnenburg, Member, IEEE, Jos Huisken, Chris Van Hoof, Member, IEEE, and Refet Firat Yazicioglu, Member, IEEE. A Configurable and Low-Power Mixed Signal SoC for Portable ECG Monitoring Applications. IEEE TRANSACTIONS ON BIOMEDICAL CIRCUITS and Systems, Vol. 8, No. 2, April 2014

[3] Alauddin Al-Omary, Wael El-Medany, Riyad Al-Hakim, "Heart Disease Monitoring System Using Web and Smartphone", IJAREEIE, Vol. 3, Issue 4, April 2014

[4] Cristian Rotariu, Hariton Costin, Dragos Arotăriți And Bogdan Dionisie, "A Wireless Ecg Module For Patient Monitoring Network", Buletinul Institutului Politehnic Din Iaşi Publicat de Universitatea Tehnică „Gheorghe Asachi” din Iaşi Tomul LIV (LVIII), Fasc. 1, 2008 Secţia Automatică şi CALCULATOARE

[5] S. Anusuya Dhandapani, Samiappan and P.R. Buvaneswari," Design and Implementation of Motion Artifact Reduction Asic for Wearable Ecg Recording", American-Eurasian Journal of Scientific Research 10 (3): 154-159, 2015 ISSN 1818-6785 (C) IDOSI Publications, 2015 DOI: 10.5829/idosi.aejsr.2015.10.3.22277

[6] R. Ezhilan and A. Balamurugan," Wearable Electrocardiogram recording using Application-specific integrated Circuit (ASIC)", Advances in Natural and Applied Sciences, 9(8) July 2015, Pages: 70-76

[7] Prakash Vidwan, Pradip Panchal, Sachin Sharma," REAL TIME PORTABLE WIRELESS ECG MONITORING SYSTEM", Asian Journal Of Computer Science And Information Technology 2: 6 (2012) $158-161$

[8] Gulab M. Dhakne, Satvashil N. Gaikwad, "A Low Power, Mixed Signal System for Wireless Transmission Of Body Temperature, Heart Beat and ECG" International Journal of Innovative Research in Computer and Communication Engineering (An ISO 3297: 2007 Certified Organization) Vol. 4, Issue 9, September 2016 